\title{
Role of diagnostic laparoscopy in chronic pelvic pain
}

\author{
Pushpa Bhatia, Pratiksha Gupta*, Devika Mor
}

Department of Gynaecology and Obstetrics, Post Graduate Institute of Medical Sciences and Researh, Basaidarapur, New Delhi, India

Received: 08 February 2016

Accepted: 05 March 2016

*Correspondence:

Dr. Pratiksha Gupta,

E-mail: drpratiksha@gmail.com

Copyright: (C) the author(s), publisher and licensee Medip Academy. This is an open-access article distributed under the terms of the Creative Commons Attribution Non-Commercial License, which permits unrestricted non-commercial use, distribution, and reproduction in any medium, provided the original work is properly cited.

\begin{abstract}
Background: Laparoscopy in chronic pelvic pain can reveal findings that cannot be detected clinically, so it can be treated and diagnosed at same sitting. This study was undertaken to evaluate role of diagnostic laparoscopy in chronic pelvic pain.

Methods: It was a prospective study conducted at department of Obstetrics and Gynecology, ESI PGIMSR, Basaidarapur, New Delhi. The study was carried out from 2012- 2014. Total 110 cases were enrolled. 55 cases who had been suffering from chronic pelvic pain for $\geq 6$ months were taken as study group (A). 55 cases without any symptoms that underwent laparoscopic sterilization were taken as control group (B). Results were statistically analyzed using Karl Pearson's correlation coefficient.

Results: Chronic pelvic pain whereas on laparoscopy normal finding were seen only in $25.45 \%$ cases. This difference was found to be statistically significance ( $\mathrm{P}$ value 0.04 ). Normal findings were seen in $60 \%$ cases of chronic pelvic pain on USG as compared to $25.14 \%$ cases on laparoscopy was statistically significant . ( p value $<0.05$.)

Conclusions: Laparoscopy is a gold standard tool in evaluation of women with chronic pelvic pain, because diagnosis and often treatment can be accomplished in one sitting, without subjecting them to exploratory laparotomy.
\end{abstract}

Keywords: Diagnostic laparoscopy, Pelvic inflammatory disease, Chronic pelvic pain

\section{INTRODUCTION}

Chronic pelvic pain (CPP) is non-cyclic pain of six or more months duration that localizes to the anatomical pelvis, anterior abdominal at or below umbilicus, the lumbosacral, and is of sufficient severity to cause functional disability or lead to medical care. ${ }^{1,2}$ Most common symptoms are dysmenorrhoea, mid-cycle pain, premenstrual tension syndrome (PMS), and endometriosis. Lower-abdominal pain can be caused by chronic pelvic inflammatory disease, pelvic adhesions, certain ovarian cysts, pelvic vascular congestion, loose support of the uterus, uterine fibroids, adenomyosis, but pain arising from organs outside pelvis may also radiate to lower abdomen.

Laparoscopy is a useful tool for the diagnosis and treatment of conditions associated with chronic pelvic pain. $^{3}$
Laparoscopy for chronic pelvic pain is based on the "see and fight" principle. ${ }^{4}$ It enables the direct inspection of intra-abdominal organs, facilitates biopsy, cultures and make therapeutic intervention possible. ${ }^{5}$ It can reveal findings that cannot be detected clinically.

\section{METHODS}

Study design Prospective study, it was conducted at department of Obstetrics and Gynecology, ESI PGIMSR, Basaidarapur, New Delhi. The study was carried out from 2012- 2014. Total of 110 cases were enrolled. 55 cases who had been suffering from chronic pelvic pain for $\geq 6$ months were taken as study group (A). 55 cases without any symptoms that underwent laparoscopic sterilization were taken as control group (B). Inclusion criteria were women age 20-40 years. Exclusion criteria were morbid obesity, severe hypertension, coronary artery disease, acute bronchiolitis, chronic obstructive lung disease, 
systemic and cardiac illness, bleeding disorders and local abdominal infection.

A written informed consent was taken from each patient. The research protocol was approved by the Institutional Ethics Committee. Patients were evaluated with detailed history. Physical examination including general physical examination, systemic and abdominal examination was done. Gynecological examination included inspection of vulva and perineum, Speculum examination of cervix and vagina, bimanual examination to assess the shape, size, direction, mobility of uterus and adnexa, mass and tenderness of urethra, vaginal fornix and cervical motion tenderness. Investigations including Complete blood count, Urine routine and microscopic, Urine culture and sensitivity, Coagulation Profile, Liver function test, Kidney function test, Chest x-ray, Electrocardiogram, transvaginal ultrasound, Cancer antigen-125, Lactic dehydrogenase, $\beta$ Human chorionic antigen, Carcino embryonic antigen were done if required.

\section{Objectives}

To study the role of diagnostic laparoscopy in chronic pelvic pain and to compare the findings of laparoscopy in patients of chronic pelvic pain and patients undergoing routine laparoscopic tubal ligation and to study the complication of laparoscopy procedure in both the groups.

\section{Laparoscopic assessment method}

Laparoscopies were performed under General anesthesia. The whole abdominal cavity was inspected including undersurface of diaphragm and liver. Further inspection was done for-Uterus, Fallopian tubes, Ovaries, Pouch of Douglas POD, Adhesion- type and site, Broad ligament, varicosity of vessel, Pelvic peritoneum. Abnormality found was recorded and treated accordingly. The observations were recorded in standard proforma for analysis. Statistical analysis was carried out using Karl Pearson's correlation coefficient. The level of significance was set as p-value less than or equal to 0.05 .

\section{RESULTS}

The mean age for the study group (Group A) was found to be 28.6 years with a range of 20- 40 years while for the control group (Group B) mean age was found to be 30.3 years with a range of 23- 40 years. Both Groups were comparable as far as age is considered (Figure 1). The incidence of chronic pelvic pain was found to be highest in Para $2(41.81 \%)$ and lowest in cases of infertility $(1.87 \%)$. In Group B, maximum number of cases were Para $2(45.45 \%)$ There was no statistically significant difference between two groups as far as literacy level was concerned. The incidence of chronic pelvic pain in Group A was found to be highest in middle class $(58.18 \%)$ and lowest in lower class (12.72\%). Similarly in Group B maximum numbers of cases were found to be in middle class $(74.54 \%)$ and lowest in lower class $(3.63 \%)$. There was no statistically significance difference between the two Groups as far as socioeconomic status was concerned. Incidence of history of previous Surgery was found to be $27.27 \%$ in Group A as compared to $38.18 \%$ in Group B (Table 1). Maximum number of cases reported with Chronic pelvic pain lasting from 6-9 months $(63.64 \%)$.Only one patient $(1.22 \%)$ reported with Pain lasting from 22 months. Mean duration of Chronic pelvic pain before laparoscopy was found to be $10.49 \pm 7.46$ months. Maximum number of cases reported with Chronic pelvic pain lasting from 6-9 months $(63.64 \%)$. Only one patient $(1.22 \%)$ reported with Pain lasting from 22 months. Mean duration of Chronic pelvic pain before laparoscopy was found to be $10.49 \pm 7.46$ months (Table 2).

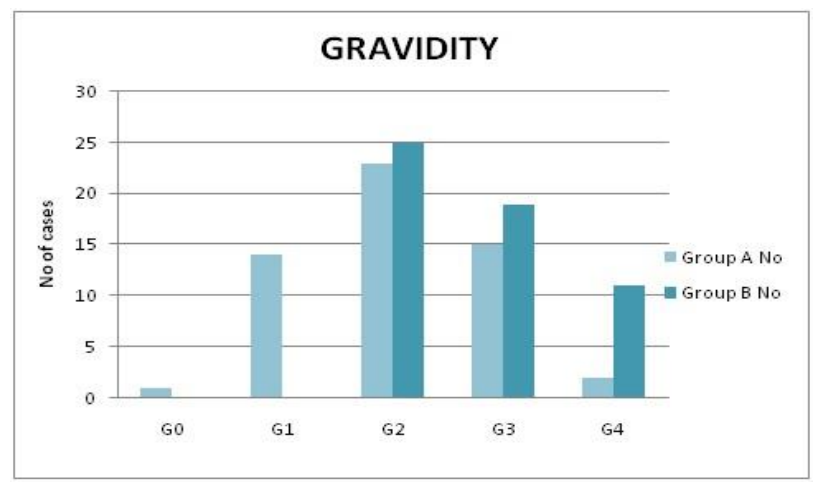

Figure 1: Distribution of cases according to gravidity.

Table 1: Distribution of cases according to age.

\begin{tabular}{|c|c|c|c|c|c|}
\hline $\begin{array}{l}\text { Age in } \\
\text { years }\end{array}$ & Group A $(\mathbf{N}=55)$ & $\begin{array}{l}\mathrm{N}=55) \\
\%\end{array}$ & \multicolumn{2}{|c|}{ Group B $(\mathrm{N}=55)$} & $\begin{array}{l}\mathrm{T} \\
\text { test }\end{array}$ \\
\hline $20-25$ & 16 & 29.09 & 07 & $12.73 \%$ & \multirow{7}{*}{0.226} \\
\hline $26-30$ & 19 & 34.55 & 23 & 41.82 & \\
\hline $31-35$ & 11 & 20.00 & 10 & 18.18 & \\
\hline $36-40$ & 09 & 16.36 & 15 & 27.27 & \\
\hline Total & 55 & 100 & 55 & 100 & \\
\hline Range & \multicolumn{2}{|c|}{$20-40$} & \multicolumn{2}{|c|}{$23-40$} & \\
\hline Mean \pm SD & \multicolumn{2}{|c|}{$28.9 \pm 6.6$} & \multicolumn{2}{|c|}{$30.3 \pm 5.5$} & \\
\hline
\end{tabular}

Table 2: Duration of pain before laparoscopy in study group (A).

\begin{tabular}{|c|c|c|}
\hline \multirow{2}{*}{$\begin{array}{l}\text { Duration of pain } \\
\text { (months) }\end{array}$} & \multicolumn{2}{|c|}{ Group A $(n=55)$} \\
\hline & No & $\%$ \\
\hline $6-9$ & 35 & 63.64 \\
\hline $10-12$ & 7 & 12.72 \\
\hline $13-15$ & 6 & 10.01 \\
\hline $16-18$ & 6 & 10.01 \\
\hline $19-22$ & 1 & 1.22 \\
\hline $16-18$ & 6 & 10.01 \\
\hline $19-22$ & 1 & 1.22 \\
\hline TOTAL & 55 & 100 \\
\hline $\begin{array}{ll}\text { Range } & 6-22 \\
\text { Mean } \pm S D & 10.49 \pm 7.46\end{array}$ & & \\
\hline
\end{tabular}


Maximum number of cases $(56.36 \%)$ had no other symptoms associated with chronic pelvic pain (Table 3 ). Associated vaginal discharge was present in $20.00 \%$ cases. One patient $(1.81 \%)$ had associated infertility. $72.72 \%$ cases with chronic pelvic pain had normal per vaginal findings (Table 4). $14.54 \%$ case had uterus mobility restricted. In $3.63 \%$ cases endometriosis and ovarian cyst was suspected was suspected whereas fibroid, to Mass and Hydrosalpinx was detected in $1.81 \%$ cases. 60\% Cases with Chronic pelvic pain had normal USG findings. $12.72 \%$ women had free fluid in POD. To mass and fibroid could be visualized in $3.63 \%$ cases. $25.25 \%$ cases of chronic pelvic pain had normal findings on laparoscopy in Group A whereas 40\%cases had normal findings in Group B. On statistical analysis this difference was found to be significant ( $\mathrm{p}$ value 0.02).74.54\% cases of Group A had abnormal findings whereas $60 \%$ cases of Group B had abnormal findings. In Study Group (A), Endometriosis found in $18.1 \%$ cases was the most common finding on laparoscopy followed by PID in $14.54 \%$ cases. Adhesions were found in $9.09 \%$ cases. All patients with adhesions had history of previous surgery. In Control Group (B) $14.54 \%$ cases had ovarian cyst, while Endometriosis was found in $7.27 \%$ cases. Adhesions were found in $7.27 \%$ cases. All cases of adhesions had history of previous surgery. Hydrosalpinx was found in $5.45 \%$ cases in both the Groups Normal per vaginal findings were found in $72.72 \%$ cases of chronic pelvic pain whereas on laparoscopy normal finding were seen only in $25.45 \%$ cases. This difference was found to be statistically significance ( $P$ value 0.04 ).PID could be diagnosed in $14.54 \%$ cases which were confirmed by laparoscopy (Table 5).

Table 3: Laparoscopic diagnosis.

\begin{tabular}{|lllll|}
\hline \multirow{2}{*}{ Laparoscopic diagnosis } & \multicolumn{2}{l}{ Group A } & \multicolumn{2}{c|}{ Group B } \\
& No & $\%$ & No & $\%$ \\
\hline Normal & 14 & 25.25 & 22 & 40 \\
\hline Endometriosis & 10 & 18.1 & 4 & 7.27 \\
\hline Adhesions & 5 & 9.09 & 4 & 7.27 \\
\hline Ovarian cyst & 4 & 7.27 & 8 & 14.54 \\
\hline To mass & 2 & 3.63 & Nil & Nil \\
\hline Fibroid & 2 & 3.63 & 2 & 3.63 \\
\hline Hydro/pyosalpinx & 3 & 5.45 & 3 & 5.45 \\
\hline PID & 8 & 14.54 & 5 & 9.09 \\
\hline Tuberculosis & 2 & 3.63 & 1 & 1.81 \\
\hline Varicosities & 1 & 1.81 & 3 & 5.45 \\
\hline $\begin{array}{l}\text { Fitz Hughes Curtis } \\
\text { Syndrome }\end{array}$ & 1 & 1.81 & 2 & 3.63 \\
\hline Endometriosis+CPI & 1 & 1.81 & 1 & 1.81 \\
\hline Fibroid+Endometriosis & 1 & 1.81 & Nil & Nil \\
\hline Adhesions+CPI & 1 & 5.45 & Nil & Nil \\
\hline
\end{tabular}

Endometriosis could be diagnosed clinically only in $3.63 \%$ cases as compared to $18.18 \%$ cases diagnosed by laparoscopy. Normal findings were seen in $60 \%$ cases of chronic pelvic pain on USG as compared to $25.14 \%$ cases on laparoscopy (Figure 2, 3, 4). This difference was found to be statistically significant ( $\mathrm{P}$ value $<0.05$.).

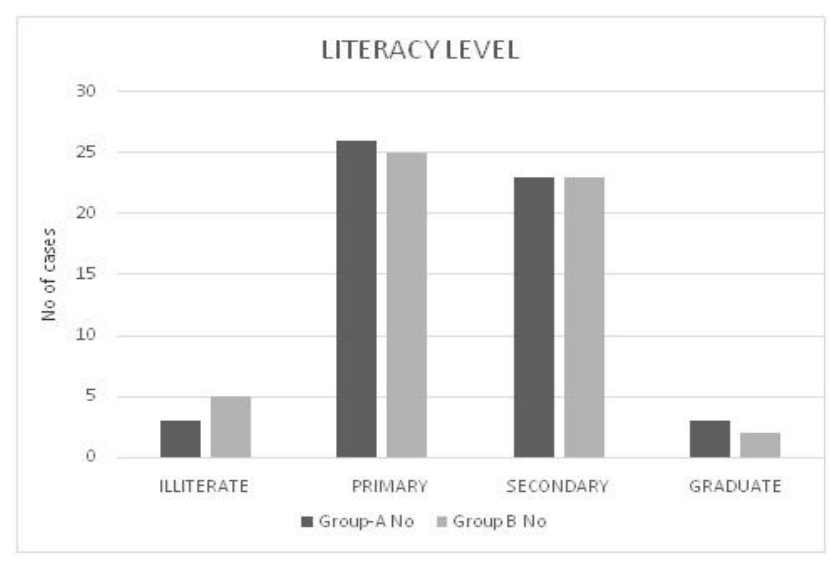

Figure 2: Distribution of cases according to literacy level.

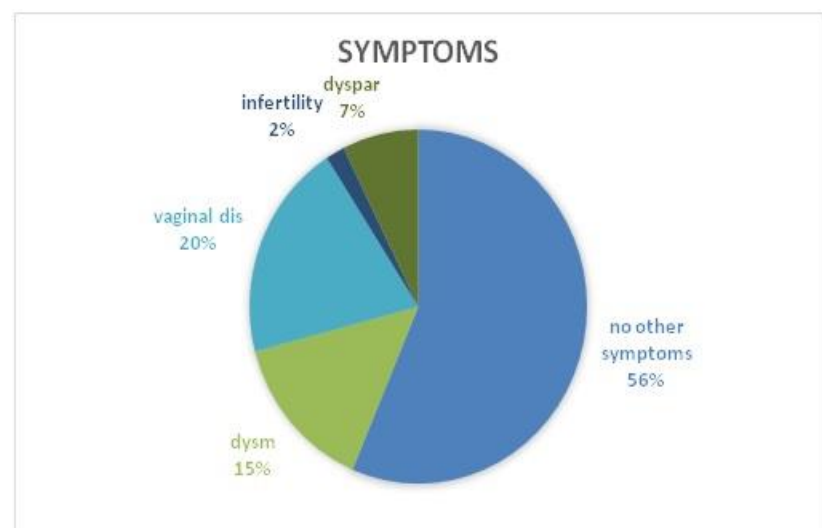

Figure 3: Other associated symptoms.

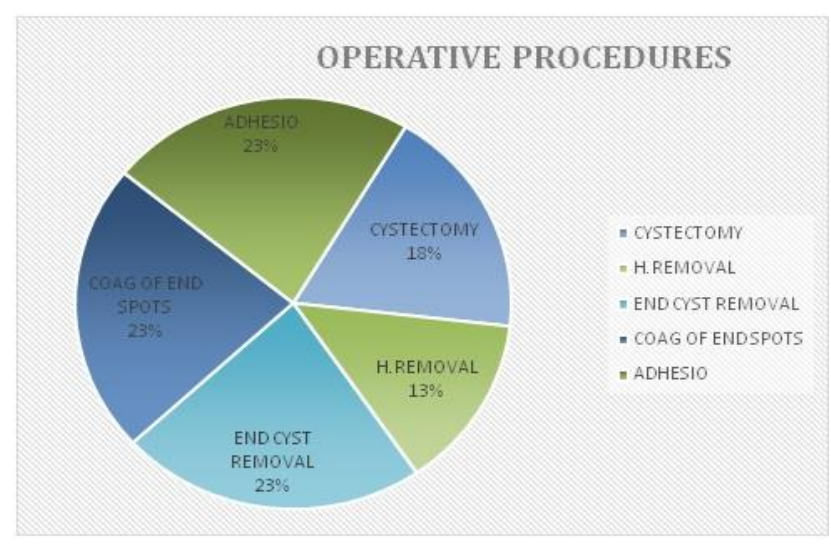

Figure 4: Operative procedures.

Endometriosis could be diagnosed only in $7.27 \%$ cases on USG as compared to $18.18 \%$ cases on laparoscopy. Extra peritoneal gas insufflations occurred in $3.63 \%$ cases of Group A and 5.45\% cases of Group B. Shoulder pain and wound infection occurred in one case each $(1.81 \%)$ in Group A. No other complications occurred in any patient in any groups. In cases of chronic pelvic pain 
adhesionlysis was done in $9.09 \%$ cases, Endometriotic cyst removal was done in $9.09 \%$ cases. Ovarian Cystectomy was done in $7.27 \%$ cases and Hydrosalpinx removal was done in $5.45 \%$ cases (Table 6).

\section{DISCUSSION}

The mean ages in our both groups were comparable. In contrast to one study there was a significant difference between two Groups in terms of Patients age.4 The incidence of CPP was found to be highest in Para 2 $(41.81 \%)$ and lowest in cases of infertility $(1.87 \%)$. In Group B, maximum number of cases were Para 2 $(45.45 \%)$. In one study cases of CPP, Parity ranged between 0-8 and nine women were Nulliparous and mean parity was 1.6 , ranging from 0 to 5.6 in present study incidence of history of previous Surgery was found to be $27.27 \%$ in Group A as compared to $38.18 \%$ in Group B. When history of previous surgery was compared to adhesions in both Groups, difference was found to be statistically significance. In one study of 86 patients 19 patients had history of previous surgery and nine had history of first trimester MTP. Eight had undergone D and E.7 and average pain duration was found to be 11.5 months.

In our study maximum number of cases reported with chronic pelvic pain lasting from 6-9 months (63.64\%). Only one patient $(1.22 \%)$ reported with Pain lasting from 22 months. Mean duration of chronic pelvic pain before laparoscopy was found to be $10.49 \pm 7.46$ months.

CPP may have other associated symptoms like dysmenorrhea, dyspareunia, abnormal uterine bleeding, infertility, vaginal discharge, etc. In present study $56.36 \%$ had no other symptoms associated. Vaginal discharge was present in $20.00 \%$ cases, $14.54 \%$ cases had dysmenorrhea and $7.27 \%$ had dyspareunia. One patient $(1.81 \%)$ had associated infertility. In one study $79.1 \%$ had acyclic abdominal pain, $26.7 \%$ had congestive dysmenorrhea. ${ }^{7}$ Cases with CPP may have normal pervaginal findings. They may have findings such as retroverted fixed uterus, restricted uterine mobility, fullness in POD, TO Mass, fornix tenderness or modularity in POD. In present study $72.72 \%$ cases with chronic pelvic pain had normal per vaginal findings. $14.54 \%$ cases had uterus mobility restricted. In $3.63 \%$ cases each endometriosis and ovarian Cyst was diagnosed, whereas fibroid, to Mass and Hydrosalpinx were detected in $1.81 \%$ cases each. In a Pelvic tenderness was found to be most common $(27.9 \%)$, followed by fornix fullness (15.1\%). 61.6\% women did not reveal any significant pathology.7 in present study $60 \%$ Cases with chronic pelvic pain had normal USG findings, $12.72 \%$ women had free fluid in POD. Adnexal cyst was present in $7.27 \%$, Hydrosalpinx was present in $5.45 \%$ cases. TO mass and fibroid could be visualized in $3.63 \%$ cases. CPP may not always be due to gynecological causes but may be due to disorders of gastrointestinal tract, urinary tract and musculoskeletal system which will not show any abnormal findings on laparoscopy. In present study
$25.25 \%$ cases of chronic pelvic pain Group A had normal findings on laparoscopy whereas $40 \%$ cases had normal findings in Control Group (B).On statistical analysis this difference was found to be significant ( $p$ value 0.02 ).

In Study group (A); an endometriosis (18.18\%) case was the most common pathological finding found on laparoscopy while $7.27 \%$ cases had Endometriosis in Control Group (B). PID was found in $14.54 \%$ cases in Study Group (A) as compared to $9.09 \%$ cases in Control Group (B).

Adhesions were found in $9.09 \%$ cases in Study Group (A), $7.27 \%$ cases in Control Group (B). All these cases had history of previous surgery which could lead to adhesions. According to a study "CPP developing from adhesions is due to restriction of bowel mobility and distension". 8 Adhesionlysis was done in all 58 cases of CPP. Ovarian cyst was more common in control Group (B)- $14.54 \%$ cases as compared to Study Group (A)$7.27 \%$ cases. All cysts were found to be simple and less than $5 \mathrm{~cm}$ in diameter. Ovarian Cystectomy was done in all cases of CPP. Fibroid and TO mass were found in $3.63 \%$ cases each in Study Group (A) whereas in Control Group (B) $3.63 \%$ cases had small fibroid and no TO mass found in Group B. Tuberculosis was detected in $3.63 \%$ cases in Study Group (A) and $1.81 \%$ cases in Control Group (B). These patients were put on ATT. Hydrosalpinx was found in $5.45 \%$ cases in both Groups. All Hydrosalpinx were removed by laparoscopy in cases of CPP. Varicosities in broad ligament were detected only in one patient in both the groups. In one study pelvic endometriosis was the most common (60.2\%). Laparoscopic finding in patients with chronic pelvic pain, followed by normal pelvic Findings in $21.2 \%$ cases and pelvic congestion in $13.0 \%$ cases. ${ }^{9}$ Other study, adhesions were found in $79.2 \%$ (n-84) of patients with chronic pelvic pain. But in Present study adhesions were found in less no of cases $(9.09 \%)$ of CPP. ${ }^{10}$

On comparing vaginal findings with laparoscopy- In present study normal per vaginal findings were present in $72.72 \%$, whereas on laparoscopy normal finding were seen in $25.45 \%$ cases. This difference was found to be statistically significant ( $\mathrm{P}$ value 0.04$)$. This shows that cause of CPP can be missed on vaginal examination and detected on laparoscopy, showing superiority of diagnostic laparoscopy over clinical examination. PID could be diagnosed in $14.54 \%$ cases clinically which was confirmed by laparoscopy. Endometriosis could be diagnosed clinically only in $3.63 \%$ cases as compared to $18.18 \%$ cases diagnosed by laparoscopy. A study also showed superiority of diagnostic laparoscopy over clinical examination as in our study. ${ }^{7}$

On comparing ultrasound findings with laparoscopy-In present study, normal findings were seen in $60 \%$ cases on USG as compared to $25.14 \%$ cases on laparoscopy. This difference was found to be statistically significant ( $p$ value 0.02). This shows that laparoscopy is better than USG for determining cause of CPP. Endometriosis could 
be diagnosed only in $7.27 \%$ cases on USG as compared to $18.18 \%$ cases on laparoscopy.

One Study revealed pelvic organ pathology in $88.4 \%$ of patients on laparoscopy as compared to $74.54 \%$ cases in present study. Their most frequent finding was endometriosis in $31.4 \%$ cases, which was much more than $18.18 \%$ cases in present study. In their study, in 36 patients Preoperative Ultrasonic examination had pelvic pathology findings and laparoscopy correlated with USG findings in $31(81.6 \%) .{ }^{6}$ Laparoscopy involves a minimal damage to body tissues. It is safer than open surgery. Although complications are rarer with laparoscopy but it can occur. In present study there was no mortality. Regarding complications of laparoscopy-In present study there was no major complication. Extra peritoneal gas insufflation occurred in $3.63 \%$ cases of Study Group (A) and $5.45 \%$ cases of Control Group (B). Shoulder pain and wound infection occurred in one case each $(1.81 \%)$ in Study Group (A). No other complications occurred in any case. In one study of 3068 cases of chronic pelvic pain, 85 complications associated with diagnostic laparoscopy were noted including 3 cases of major complications. Major complications included bowel perforation and bowel herniation through the umbilical trocar site, and all 3 patients required immediate correction. Minor complications included wound infection, abdominal discomfort after laparoscopy..$^{9,11}$

Laparoscopy is also called minimal invasive surgery. Various small operative procedures can be done from laparoscope. Laparoscopy surgery causes reduced pain and short recovery time. In present study adhesionlysis was done in $9.09 \%$ cases, Endometriotic cyst removal in $9.09 \%$, Ovarian Cystectomy in $7.27 \%$ cases and Hydrosalpinx removal was done in $5.45 \%$ cases. In one study of 44 patients adhesionlysis and ovarian cyst removal was done in 8 , endometrioma excision was done In 9 and salpingectomy and endometrioma cauterization was done in one patient each. ${ }^{4}$ Other studies have also shown almost similar results and interesting findings. ${ }^{12,13 \text {, }}$ 14, ${ }^{15}$ limitation. Thus our study concludes that laparoscopy is an excellent tool in evaluation of women with chronic pelvic pain, because diagnosis and often treatment can be accomplished in one sitting, without subjecting them to exploratory laparotomy.

\section{CONCLUSIONS}

Laparoscopy is a gold standard tool in evaluation of women with chronic pelvic pain, because diagnosis and often treatment can be accomplished in one sitting, without subjecting them to exploratory laparotomy.

\section{ACKNOWLEDGMENTS}

I am grateful to the operation theatre staff and financial and material support by institutional fund.
Funding: No funding sources

Conflict of interest: None declared

Ethical approval: The study was approved by the Institutional Ethics Committee

\section{REFERENCES}

1. Howard FM. The role of laparoscopy as a diagnostic tool in chronic pelvic pain. Baillieres Best Pract Res Clin Obstet Gynaecol. 2000;14(3):467-94.

2. Papathanasiou K, Papageorgiou C, Panidis D, Mantalenakis S. Our experience in laparoscopic diagnosis and management in women with chronic pelvic pain. Clin Exp Obstet Gynecol. 1999;26(34):190-2.

3. Lamvu G, Tu F, As-Sanie S, Zolnoun D, Steege JF. The role of laparoscopy in the diagnosis and treatment of conditions associated with chronic pelvic pain. Obstet Gynecol Clin North Am. 2004;31(3):619-30.

4. Demir F, Ozcimen EE, Oral HB. The role of gynecological, urological, and psychiatric factors in chronic pelvic pain. Arch Gynecol Obstet. 2012;286(5):1215-20.

5. Renaer M. Chronic pelvic pain in women, p l. New York:Springer-Verlag. 1981.

6. Zúbor P, Szunyogh N, Galo S, Biringer K, Dókus K, Visnovský J. [Laparoscopy in chronic pelvic pain--a prospective clinical study]. Ceska Gynekol. 2005;70(3):225-31.

7. Hebbar S, Chawla C. Role of laparoscopy in evaluation of chronic pelvic pain. J Minim Access Surg. 2005;1(3):116-20.

8. Duffy DM, diZerega GS. Adhesion controversies: pelvic pain as a cause of adhesions, crystalloids in preventing them. J Reprod Med. 1996;41(1):19-26.

9. Kang SB, Chung HH, Lee HP, Lee JY, Chang YS. Impact of diagnostic laparoscopy on the management of chronic pelvic pain. Surg Endosc. 2007;21(6):916-9.

10. Mettler L, Alhujeily M. Role of laparoscopy in identifying the clinical significance and cause of adhesions and chronic pelvic pain: a retrospective review at the Kiel School of Gynecological Endoscopy. JSLS. 2007;11(3):303-8.

11. Fuller J, Ashar BS, Carey-Corrado J. Trocarassociated injuries and fatalities: an analysis of 1399 reports to the FDA. J Minim Invasive Gynecol. 2005;12(4):302-7.

12. Gogate A, Brabin L, Nicholas S, Gogate S, Gaonkar T, Naidu A, et al. Risk factors for laparoscopically confirmed pelvic inflammatory disease: findings from Mumbai (Bombay), India. Sex Transm Infect. 1998;74(6):426-32.

13. Eftekhar M, Soheila P, Zadeh LM. Coexisting pelvic tuberculosis and endometriosis presenting in an infertile woman: Report of a rare case. Iran J Reprod Med. 2014;12(6):439-41.

14. Avan BI, Fatmi Z, Rashid S. Comparison of clinical and laparascopic features of infertile women 
suffering from genital tuberculosis (TB) or pelvic inflammatory disease (PID) or endometriosis. J Pak Med Assoc. 2001;51(11):393-9.

15. Jaiyeoba O, Soper DE. A practical approach to the diagnosis of pelvic inflammatory disease. Infect Dis Obstet Gynecol. 2011;2011:753037.
Cite this article as: Bhatia $\mathrm{P}$, Gupta $\mathrm{P}$, Mor D. Role of diagnostic laparoscopy in chronic pelvic pain. Int J Reprod Contracept Obstet Gynecol 2016;5:1152-7. 